\title{
FITTING CLASSES BASED ON GROUPS OF NILPOTENT LENGTH THREE WITH OPERATOR-ISOMORPHIC MINIMAL NORMAL SUBGROUPS
}

\author{
BRENDAN MCCANN
}

(Received 12 October 1989)

Communicated by $\mathrm{H}$. Lausch

\begin{abstract}
In this paper a technique for constructing Fitting Classes is applied to certain groups of nilpotent length three which have non-unique minimal normal subgroups. A characterisation of the minimal Fitting Class of some of these groups is also given.
\end{abstract}

1991 Mathematics subject classification (Amer. Math. Soc.) 20 D 35, 20 D 10.

Some recent work on minimal Fitting classes deals with groups of nilpotent length three or more which are monolithic, that is, which have unique minimal normal subgroups (see Bryce [5], Bryce, Cossey and Ormerod [6] and McCann [2], [3] and [4]). The study of Fitting classes based on monolithic groups goes back to Dark [7], a paper to which the above are indebted.

This paper applies methods of Fitting class construction, which are similar to those in the above papers, to certain non-monolithic groups. The groups in question are of nilpotent length three and have non-unique minimal normal subgroups which are operator-isomorphic with respect to conjugation. They are semi-direct products of elementary abelian $r$-groups (where $r$ is prime) by certain $r^{\prime}$-groups, given in matrix form. Facts about general linear groups, especially Lemma 1.4, will play an important role in this paper. We note here that all groups dealt with will be finite.

In Section 1 we define the groups on which the Fitting classes will be based. Some basic results from the theory of linear groups will also be given.

(C) 1991 Australian Mathematical Society $0263-6115 / 91 \$ A 2.00+0.00$ 
Section 2 deals with the "basic" Fitting class construction, while in Section 3 we look at the minimal Fitting class of an even more restricted type of (non-monolithic) group. The final section gives some results which indicate why the groups dealt with have been so restrictively chosen.

I would like to thank an Roinn Oideachais for the support of a postdoctoral fellowship while doing some of the research contained in this paper, as well as the Mathematics Departments of Coláisti na hOllscoile, Gaillimh agus Corcaigh. I would also like to thank Professor Hermann Heineken for his hospitality at Universität Würzburg in July/August 1988.

\section{Notation and preliminary results}

The notation and conventions of this section will be used throughout the rest of this paper. We let $p, q$ and $r$ be primes and $\alpha, m$ and $t$ be natural numbers which satisfy:

(i) $p \neq 2, p \neq r$;

(ii) $q \neq r$;

(iii) $p \mid(q-1)$;

(iv) $p \mid t$;

(v) $t$ is the minimal natural number such that $q^{\alpha} \mid\left(r^{t}-1\right)$;

(vi) $1 \leq m<p$.

We let $F$ be the field with $r^{t}$ elements and denote the additive group of $F$ by $U$. Thus $U$ can be considered as a vector space of dimension $t$ over $Z_{r}$ (the field of order $r$, that is, the prime field of $F$ ), or as an elementary abelian $r$-group of rank $t$. We let the multiplicative group of $F$ be generated by $\gamma_{1}$, say.

By the theory of finite fields the automorphism group of $F$ is cyclic of order $t$, so by (iv) there is an element, $\delta_{1}$, of order $p$ in $\operatorname{Aut}(F)$. Multiplication by $\gamma_{1}$ in $F$ induces an invertible linear transformation on $U$, as does the "natural" action of $\delta_{1}$. We identify $\operatorname{Aut}(U)$ with $\mathrm{GL}(t, r)$, the general linear group of invertible $t \times t$ matrices over $Z_{r}$. In addition we identify $\gamma_{1}$ and $\delta_{1}$ with the transformations induced by them on $U$. So we consider $\gamma_{1}$ and $\delta_{1}$ as $t \times t$ matrices over $Z_{r}$ (relative to some fixed basis of $U$ ). By (v) there exists an element $\omega_{1} \in\left\langle\gamma_{1}\right\rangle$ such that $o\left(\omega_{1}\right)=q^{\alpha}$, and, also by (v), $U$ is irreducible under $\left\langle\omega_{1}\right\rangle$. It may be seen that $\delta_{1}$ normalises $\left\langle\gamma_{1}\right\rangle$ and hence also $\left\langle\omega_{1}\right\rangle$, but $\delta_{1}$ does not centralize either of these two groups.

In order to construct groups with operator-isomorphic minimal normal subgroups we take the tensor-product of the above representation of $\left\langle\gamma_{1}, \delta_{1}\right\rangle$ with the identity representation of suitable degree. Since we shall be dealing 
directly with general linear groups, we define the tensor-product in terms of matrices. Namely we define the $m t \times m t$ matrices (when $m$ is as in (vi)) $\gamma, \omega$ and $\delta$ by:

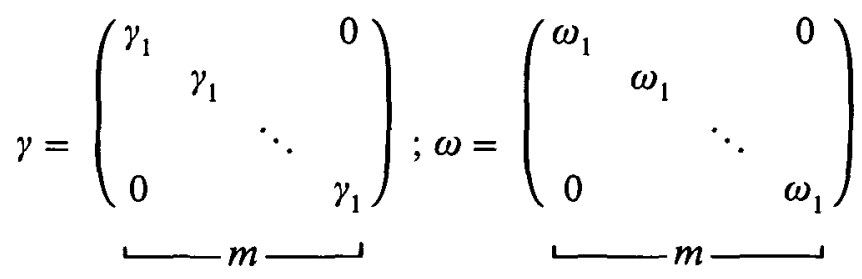

and

$$
\delta=\left(\begin{array}{llll}
\delta_{1} & & & 0 \\
& \delta_{1} & & \\
& & \ddots & \\
0 & & & \delta_{1}
\end{array}\right)
$$

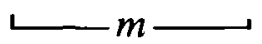

That is $\gamma, \omega$ and $\delta$ have $t \times t$ blocks, equal to $\gamma_{1}, \omega_{1}$ and $\delta_{1}$ respectively, along the main diagonal and zero entries elsewhere.

We define $V$ to be the direct sum $V=V_{1} \oplus V_{2} \oplus \cdots \oplus V_{m}$, where, for $i=1, \ldots, m, V_{i} \cong U$. We let $\langle\gamma, \delta\rangle$ operate on $V$ with the "natural" action, that is $V_{1}, \ldots, V_{m}$ are operator-isomorphic $\langle\gamma, \delta\rangle$-submodules of $V$ and $\langle\gamma, \delta\rangle$ acts on $V_{i}$ as $\left\langle\gamma_{1}, \delta_{1}\right\rangle$ on $U$ (for $i=1, \ldots, m$ ).

For ease of notation we further define $K$ to be the class of groups which satisfy: $K \cong V \rtimes\langle\omega, \delta\rangle$, where $V$ and $\langle\omega, \delta\rangle$ are defined as above for some suitable $p, q, r, \alpha, m$ and $t$ which satisfy (i), $\ldots$, (vi). We will construct Fitting classes to show that if $K_{1}$ and $K_{2}$ are elements of $\mathbf{K}$, then $K_{1} \in \operatorname{Fit}\left(K_{2}\right)$, the minimal Fitting class containing $K_{2}$, if and only if $K_{1} \cong$ $K_{2}$. To construct these classes we take some fixed $\langle\omega, \delta\rangle$ and $V$, identify $\operatorname{Aut}(V)$ with $\mathrm{GL}(m t, r)$ and examine the centralizers of certain subgroups which have $\omega$ as an element in $\operatorname{GL}(m t, r)$. The following notation will be used:

$$
\begin{aligned}
C & =C_{\mathrm{GL}(m t, r)}(\omega) ; \\
N & =N_{\mathrm{GL}(m t, r)}(\langle\omega\rangle) .
\end{aligned}
$$

Note that since $\langle\omega\rangle$ is a characteristic subgroup of $\langle\omega, \delta\rangle$, we have

$$
N_{\mathrm{GL}(m t, r)}(\langle\omega, \delta\rangle) \leq N,
$$

and $C_{\mathrm{GL}(m t, r)}(\langle\omega, \delta\rangle) \leq C$. We also note the following elementary result from linear algebra. 
LEMMA 1.1. Let $F$ be the field with $r^{t}$ elements. Then $C \cong \mathrm{GL}(m, F)$.

Proof. Let $g \in \mathrm{GL}(m t, r)$. Then we can write $g$ as

$$
g=\left(\begin{array}{ccc}
g_{11} & \cdots & g_{1 m} \\
\vdots & & \vdots \\
g_{m 1} & \cdots & g_{m m}
\end{array}\right)
$$

where the $g_{i j}$ are $t \times t$ block matrices, $(i, j=1, \ldots, m)$. We then have $g \omega=\omega g$ if and only if

$$
\left(\begin{array}{ccc}
g_{11} \omega_{1} & \cdots & g_{1 m} \omega_{1} \\
\vdots & & \vdots \\
g_{m 1} \omega_{1} & \cdots & g_{m m} \omega_{1}
\end{array}\right)=\left(\begin{array}{ccc}
\omega_{1} g_{11} & \cdots & \omega_{1} g_{1 m} \\
\vdots & & \vdots \\
\omega_{1} g_{m 1} & \cdots & \omega_{1} g_{m m}
\end{array}\right) .
$$

So we see that $\omega_{1}$ commutes with all of the $g_{i j}$. An application of Schur's lemma (Huppert $[1, \mathrm{I}, 10.5])$ shows that $C_{\operatorname{End}(U)}\left(\omega_{1}\right) \cong F$. Thus $C$ is isomorphic to the group of invertible $m \times m$ matrices over $F$.

Lemma 1.1 is useful in that it allows us to calculate the order of a Sylow $p$-subgroup of $C$ from that of $\operatorname{GL}(m, F)$. Before we determine a particular Sylow $p$-subgroup of $C$ we need some more notation. We define $s$ to be the least non-negative integer such that $p \mid\left(r^{s t}-1\right)$, and we define $\beta$ by means of $p^{\beta} T\left(r^{s t}-1\right)$. Here the symbol T stands for "the largest power of $p$ to divide". For our fixed $m$, as in (vi), we let $m=s k+\varepsilon$, for a suitable $k$ and $\varepsilon$, with $0 \leq \varepsilon<s$. We will retain this definition of $s, \beta, k$ and $\varepsilon$ in what follows.

We let

$$
W_{i}=V_{s(i-1)+1} \oplus \cdots \oplus V_{s i}, \text { for } i=1, \ldots, k,
$$

and consider restrictions of $\langle\gamma, \delta\rangle$ to $W_{1}$, say. We define $F_{r^{s t}}$ to be the field of order $r^{s t}$ and, as $W_{1}$ has order $r^{s t}$, we consider $W_{1}$ to be the additive group of $F_{r^{t}}$. Now, $F_{r^{s t}}$ has a subfield of order $r^{t}$ so we embed $F$ (as above) in $F_{r^{s t}}$. In particular we have $\langle\gamma\rangle \leq F_{r^{s t}}^{\times}$, the multiplicative group of $F_{r^{s t}}$. We let $F_{r^{s t}}^{\times}$operate by multiplication on $W_{1}$, and can check that, relative to a suitable basis, this action of $\gamma$ on $W_{1}$ is given in $s t \times s t$ matrix form as

$$
\gamma \sim\left(\begin{array}{cccc}
\gamma_{1} & & & 0 \\
& \gamma_{1} & & \\
& & \ddots & \\
0 & & & \gamma_{1}
\end{array}\right)
$$

(where we identify $\operatorname{Aut}\left(W_{1}\right)$ with GL$(s t, r)$ ). By the definition of $\beta$ and $s$, we have $p^{\beta} T\left|F_{r^{s t}}^{\times}\right|$, so, since $F_{r^{t}}^{\times}$cyclic, there is an element, $h$, of order 
$p^{\beta}$ in $\operatorname{Aut}\left(W_{1}\right)$ which centralises $\gamma$ and thus also $\omega$. We assume $h$ to be given in $s t \times s t$ matrix from (relative to the above basis of $W_{1}$ ).

We let $C_{1}=C_{\mathrm{Aut}\left(W_{1}\right)}(\omega)$. By Lemma 1.1 we have $C_{1} \cong \mathrm{GL}(s, F)$. Now we have $|\mathrm{GL}(s, F)|=\left(r^{s t}-1\right)\left(r^{s t}-r^{t}\right) \cdots\left(r^{s t}-r^{(s-1) t}\right)$, so we see that, by the definition of $s$ and $\beta, p^{\beta} T\left|C_{1}\right|$. If we consider $\delta$ to be restricted to $W_{1}$ (that is, we consider $\delta$ to be the st $\times$ st matrix:

$$
\delta \sim\left(\begin{array}{cccc}
\delta_{1} & & & 0 \\
& \delta_{1} & & \\
& & \ddots & \\
0 & & & \delta_{1}
\end{array}\right),
$$

we see by Sylow's theorems that $\delta$ normalises some Sylow $p$-subgroup of $C_{1}$ (this because $\delta \in N$ ). Since, by comparison of orders, $\langle h\rangle$ is a Sylow $p$-subgroup of $C_{1}$, we may assume that, as matrices, $\delta$ normalises $\langle h\rangle$.

We define the $m t \times m t$ matrices $h_{i}, i=1, \ldots, k$, as follows

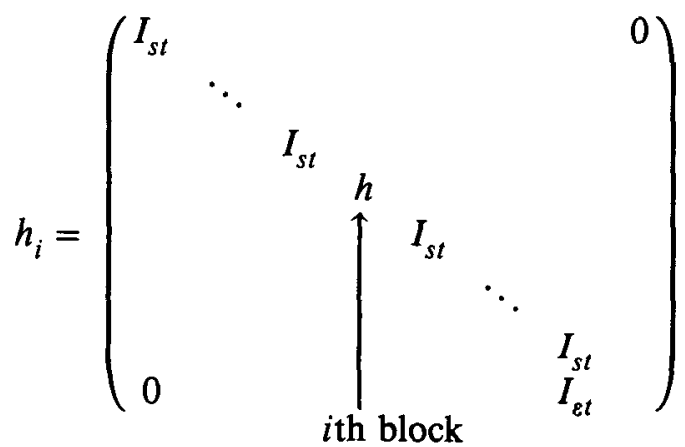

(where $I_{n}$ is an $n \times n$ identity matrix for the natural number $n$ ). Then $\left\langle h_{1}, \ldots, h_{k}\right\rangle=\left\langle h_{1}\right\rangle \times \cdots \times\left\langle h_{k}\right\rangle \cong C_{p^{\beta}} \times \cdots \times C_{p^{\beta}}$.

In addition, by the above identifications, we have $\left\langle h_{1}, \ldots, h_{k}\right\rangle \leq C$ and $\delta$ (considered once more as an $m t \times m t$ matrix) normalises each one of $\left\langle h_{1}\right\rangle, \ldots,\left\langle h_{k}\right\rangle$. We let $P=\left\langle h_{1}, \ldots, h_{k}\right\rangle$. Since $\langle\omega\rangle$ is cyclic of order $q^{\alpha}$ and $q$ is a prime with (by (iii) say) $q \neq 2, N / C$ is cyclic. Thus $\langle\delta\rangle C / C$ is the unique subgroup of order $p$ in $N / C$. The following result tells us something about $\langle\delta\rangle P$.

LEMMA 1.2. $\langle\delta\rangle P$ is a Sylow p-subgroup of $\langle\delta\rangle C$.

Proof. We need only show that $P$ is a Sylow $p$-subgroup of $C$. Since $P$ has order $p^{k \beta}$, we must show, by Lemma 1.1 , that $p^{k \beta} \mathrm{T}|\mathrm{GL}(m, F)|$. Note that the trivial case where $s>m$ (whence $k=0$ ) is covered by taking 
$P=1$. Now

$$
\begin{aligned}
|\mathrm{GL}(m, F)| & =\left(r^{t m}-1\right)\left(r^{t m}-r^{t}\right) \cdots\left(r^{t m}-r^{t(m-1)}\right) \\
& =\left(r^{t m}-1\right)\left(r^{t(m-1)}-1\right) \cdots\left(r^{t}-1\right) r^{m(m-1) t / 2} .
\end{aligned}
$$

Let $n$ be such that $1 \leq n \leq m$. Then $n=s d+e$, for suitable values $d$ and $e$ with $0 \leq e<s$. We can see that if $p$ divides $r^{n t}-1$, then $p$ divides $r^{e t}-1$. By the choice of $s$, this implies that $p \mid\left(r^{n t}-1\right)$ if and only if $n$ is a multiple of $s$.

Suppose now that $n=f s \leq m$, for some suitable $f$. We show that $p^{\beta} T\left(r^{n t}-1\right)$. We have

$$
r^{n t}-1=r^{t s f}-1=\left(r^{t s}-1\right)\left(r^{t s(f-1)}+\cdots+r^{t s}+1\right) .
$$

Since $r^{t s} \equiv 1 \bmod \left(p^{\beta}\right)$, we have

$$
\begin{aligned}
r^{t s(f-1)}+\cdots+r^{t s}+1 \equiv & 1+\cdots+1 \bmod \left(p^{\beta}\right) \\
& \llcorner f \text { times } \uparrow \\
& \equiv f \bmod \left(p^{\beta}\right) .
\end{aligned}
$$

But $f \leq m<p$. Thus $p$ does not divide $r^{t s(f-1)}+\cdots+r^{t s}+1$, so we conclude $p^{\beta} T\left(r^{n t}-1\right)$.

There are exactly $k$ distinct values of $n$ with $s \mid n$ and $0<n \leq m$. We thus have $p^{k \beta}|\mathrm{GL}(m, F)|$.

We note that $W_{1}, \ldots, W_{k}, V_{s k+1}, \ldots, V_{s k+\varepsilon}$ are all invariant under $\langle\omega, \delta, P\rangle$. The next lemma shows how $W_{i}$ may be decomposed under certain subgroups of $\langle\omega, P\rangle$.

LEMMA 1.3. Let $1 \neq x \in P$. Then either or

(i) $W_{i}$ is irreducible under $\langle x, \omega\rangle$

(ii) $x$ centralises $W_{i}$ and, in particular, $V_{s(i-1)+1}, \ldots, V_{s i}$ are all invariant under $\langle x, \omega\rangle$.

Proof. If $s=1$ then $W_{i}=V_{i}$ and we are done. Now assume that $s>1$ and that $W_{i}$ is not irreducible under $\langle x, \omega\rangle$. In addition we assume, without loss of generality, that $C_{\langle x\rangle}\left(W_{i}\right)=1$. Thus $\langle x, \omega\rangle$ is an $r^{\prime}$-group of automorphisms of $W_{i}$. We apply Maschke's Theorem to find a subgroup, $T$, of $W_{i}$ such that $T$ is irreducible under $\langle x, \omega\rangle$ and that $C_{\langle x\rangle}(T)=1$. Since $T$ is invariant under $\langle\omega\rangle$, we see that $|T|=r^{d t}$, for some $d$ with $1 \leq d\langle s$. Now $\langle x, \omega\rangle$ is abelian so, in particular, $\langle x\rangle$ must act fixpoint freely on $T$. Thus $o(x) \mid(|T|-1)$, whence we have $p \mid\left(r^{d t}-1\right)$, which contradicts our choice of $s$. We conclude that $x$ centralises $W_{i}$. 
MaIn Lemma 1.4. Let $g$ be a p-element of $\langle\delta\rangle C$ which does not centralize $\omega$. Then $C_{\mathrm{Aut}(V)}(\langle g, \omega\rangle)$ is isomorphic to a subgroup of $\mathrm{GL}\left(m, F_{r^{n}}\right)$, where $F_{r^{n}}$ is the field with $r^{n}$ elements, and $n$ is such that $t=n p$.

Proof. We demonstrate the result first in the case where there is a decomposition $V=U_{1} \oplus \cdots \oplus U_{m}$, where $U_{j}$ is a faithful irreducible $\langle\omega\rangle$ submodule, which is invariant under $\langle g, \omega\rangle$, for $j=1, \ldots, m$.

By the definition of $V$ and $\langle\omega\rangle$ we see that all the $U_{j}$ are operatorisomorphic to $V_{1}$ with respect to $\langle\omega\rangle$. We assume that $U_{1}, \ldots, U_{d}$ are all $\langle g, \omega\rangle$-isomorphic but that $U_{1}$ is not $\langle g, \omega\rangle$-isomorphic to any of $U_{d+1}, \ldots$, $U_{m}$. Let $c \in C_{\operatorname{Aut}(V)}(\langle g, \omega\rangle)$. Then for any $j, U_{j}^{c}$ is $\langle g, \omega\rangle$-isomorphic to $U_{j}$ and we infer that $c$ normalises the groups $S=U_{1} \oplus \cdots \oplus U_{d}$ and $T=U_{d+1} \oplus \cdots \oplus U_{m}$. We identify $\operatorname{Aut}(S)$ and $\operatorname{Aut}(T)$ with the "natural" subgroups of $\operatorname{Aut}(V)$. Thus

$$
C_{\mathrm{Aut}(V)}(\langle g, \omega\rangle)=C_{\mathrm{Aut}(S)}(\langle g, \omega\rangle) \times C_{\mathrm{Aut}(T)}(\langle g, \omega\rangle) .
$$

By considering the restriction of $\langle g, \omega\rangle$ to $U_{1}$ and identifying $U_{1}$ with the additive group of $F$, the field with $r^{t}\left(=r^{n p}\right)$ elements, we see, using say [3, III.3], that $C_{\operatorname{End}\left(U_{1}\right)}(\langle g, \omega\rangle)$ is isomorphic to $F_{r^{n}}$, the subfield of $F$ formed by those elements fixed by the Galois automorphism of order $p$. As in Lemma 1.1 we have $C_{\mathrm{Aut}(S)}(\langle g, \omega\rangle) \cong \mathrm{GL}\left(d, F_{r^{n}}\right)$. We assume inductively that $C_{\mathrm{Aut}(T)}(\langle g, \omega\rangle) \tilde{\leq} \mathrm{GL}\left(m-d, F_{r^{n}}\right)$ and conclude that

$$
\begin{aligned}
C_{\mathrm{Aut}(V)}(\langle g, \omega\rangle) & =C_{\mathrm{Aut}(S)}(\langle g, \omega\rangle) \times C_{\operatorname{Aut}(T)}(\langle g, \omega\rangle), \\
& \leq \mathrm{GL}\left(d, F_{r^{n}}\right) \times \mathrm{GL}\left(m-d, F_{r^{n}}\right) \\
& \simeq \mathrm{GL}\left(m, F_{r^{n}}\right) .
\end{aligned}
$$

For the general case we may assume, by Sylow's theorems, that $g \in$ $\langle\delta\rangle P$. Then $W_{1}, \ldots, W_{k}, V_{s k+1}, \ldots, V_{m}$ are all invariant under $\langle g, \omega\rangle$. If none of $W_{1}, \ldots, W_{k}$ is irreducible under $\left\langle g^{p}, \omega\right\rangle$, then by Lemma 1.3 $g^{p}$ centralises $W_{1}, \ldots, W_{k}$ (since $g^{p} \in P$ ). Since $P$ already centralises $V_{s k+1}, \ldots, V_{m}$, we see that $g^{p}=1$, that is, $o(g)=p$. Now let $U$ be a submodule of $W_{i}$ which is irreducible under $\langle g, \omega\rangle$. By a result of Zassenhaus (see, for example [2, II.5] for a proof), either $U$ is irreducible under $\langle\omega\rangle$ or $U$ is the direct sum of $p\langle\omega\rangle$-submodules. In the latter case, since all $\langle\omega\rangle$-submodules of $V$ have order greater than or equal to $r^{t}$, the contradiction $|U| \geq r^{p t}>r^{m t}=|V|$, would arise. Thus, applying Maschke's Theorem, we find a decomposition $W_{i}=U_{i_{1}} \oplus \cdots \oplus U_{i_{s}}$ for $i=1, \ldots, k$, where the $U_{i_{j}}$ are invariant under $\langle g, \omega\rangle$ and are irreducible under $\langle\omega\rangle$. This brings 
us back to the case already dealt with, so we now assume that $W_{1}$, say, is irreducible under $\left\langle g^{p}, \omega\right\rangle$ (and so also under $\langle g, \omega\rangle$ ).

We assume an enumeration such that $W_{1}, \ldots, W_{d}$ are operator-isomorphic with regard to $\langle g, \omega\rangle$, for some $d \leq k$, but that $W_{1}$ is not operatorisomorphic to any of $W_{d+1}, \ldots, W_{k}, V_{s k+1}, \ldots, V_{m}$. As above, we let $S=W_{1} \oplus \cdots \oplus W_{d}$ and $T=W_{d+1} \oplus \cdots \oplus W_{k} \oplus V_{s k+1} \oplus \cdots \oplus V_{m}$ and have

$$
C_{\mathrm{Aut}(V)}(\langle g, \omega\rangle)=C_{\mathrm{Aut}(S)}(\langle g, \omega\rangle) \times C_{\mathrm{Aut}(T)}(\langle g, \omega\rangle) .
$$

For notational convenience, we assume $C_{\langle g\rangle}\left(W_{1}\right)=1$. We let $o(g)=p^{f+1}$ and let $x=g^{p}$, so $x w$ has order $p^{f} q^{\alpha}$. In addition we let $F_{r^{s t}}$ be the field with $r^{s t}$ elements, identify $W_{1}$ with the additive group of $F_{r^{s t}}$ and $x \omega$ with an element of order $p^{f} q^{\alpha}$ in the multiplicative group of $F_{r^{s t}}$. Applying Schur's Lemma as in Lemma 1.1, we see that $C_{\operatorname{End}\left(W_{1}\right)}(x \omega)$ is isomorphic to $F_{r^{s t}}$. As above we let $F_{r^{s n}}$ be the subfield of order $r^{s n}$ in $F_{r^{s t}}$ which comprises those elements fixed by the Galois automorphism of order $p$ and see that

$$
C_{\mathrm{End}\left(W_{1}\right)}(\langle g, \omega\rangle)=C_{\mathrm{End}\left(W_{1}\right)}(\langle x \omega, \delta\rangle) \cong F_{r^{s^{n}}}
$$

Since $W_{1}, \ldots, W_{d}$ are $\langle g, \omega\rangle$-isomorphic, we see, as in Lemma 1.1, that

$$
C_{\mathrm{Aut}(S)}(\langle g, \omega\rangle) \cong \mathrm{GL}\left(d, F_{r^{s}}\right) .
$$

Since $F_{r^{n}}$ can be identified with a subfield of $F_{r^{s n}}$, we see that $\operatorname{GL}\left(d, F_{r^{s n}}\right)$ can be embedded in $\operatorname{GL}\left(s d, F_{r^{n}}\right)$. We again inductively assume that the group $C_{\mathrm{Aut}(T)}(\langle g, \omega\rangle)$ can be embedded in $\mathrm{GL}\left(m-s d, F_{r^{n}}\right)$, whence

$$
\begin{aligned}
C_{\mathrm{Aut}(V)}(\langle g, \omega\rangle) & =C_{\mathrm{Aut}(S)}(\langle g, \omega\rangle) \times C_{\mathrm{Aut}(T)}(\langle g, \omega\rangle) \\
& \simeq \mathrm{GL}\left(s d, F_{r^{n}}\right) \times \mathrm{GL}\left(m-s d, F_{r^{n}}\right) \\
& \simeq \mathrm{GL}\left(m, F_{r^{n}}\right) .
\end{aligned}
$$

COROLlaRy 1.5. Let $g$ be as in Lemma 1.4. Then there exists no element in $C_{\mathrm{Aut}(V)}(\langle g, \omega\rangle)$ which satisfies $o(y)=q^{\alpha}$.

Proof. By Lemma 1.4 $C_{\mathrm{Aut}(V)}(\langle g, \omega\rangle)$ is isomorphic to a subgroup of $\mathrm{GL}\left(m, F_{r^{n}}\right)$. But $\mathrm{GL}\left(m, F_{r^{n}}\right)$ can be embedded as a subgroup of $\mathrm{GL}(m n, r)$. If there exists an element $y$ of $\mathrm{GL}(m n, r)$ with $o(y)=q^{\alpha}$, then we see that $q^{\alpha} \mid\left(r^{e}-1\right)$ for some $e$ with $e \leq m n$. But $m n<p n=t$ and we have a contraction to condition (v). 


\section{A Fitting class construction}

For this section we let $K=V \rtimes\langle\omega, \delta\rangle$ be a fuxed element of $\mathbf{K}$ relative to the fixed primes and natural numbers $p, q, r, \alpha, m$ and $t$ which satisfy (i) , .., (vi) of Section 1 . We recall that a Fitting class, $\mathbf{F}$, is a set of groups with the following properties:

1. If $G$ is an element of $\mathbf{F}$, then so is every isomorphic copy of $G$;

2. If $G_{1} \unlhd G \in \mathbf{F}$, then $G_{1} \in \mathbf{F}$;

3. If $G=G_{1} G_{2}$ with $G_{j} \unlhd G$ and $G_{j} \in \mathbf{F}$, for $j=1,2$, then $G \in \mathbf{F}$.

For the set of primes $\pi$ we also recall that the $\pi$-residual of the group $G$ is

$$
0^{\pi}(G)=\bigcap_{N \unlhd G, G / N \text { a } \pi \text {-group }} N
$$

and the $\pi$-radical of $G$ is

$$
\left.0_{\pi}(G)=\langle N| N \unlhd G, N \text { is a } \pi \text {-group }\right\rangle .
$$

(We use $p$ to denote the set $\{p\}$, where $p$ is a prime $-p^{\prime}$ denotes the set of all primes except $p$.) Note that if $G_{1} \unlhd G$, then $0^{\pi}\left(G_{1}\right) \leq 0^{\pi}(G)$ and $0_{\pi}\left(G_{1}\right)=G_{1} \cap 0_{\pi}(G)$, while if $G=G_{1} G_{2}$ where $G_{1}$ and $G_{2}$ are normal in $G$, then $0^{\pi}(G)=0^{\pi}\left(G_{1}\right) 0^{\pi}\left(G_{2}\right)$.

In order to construct a "non-trivial" Fitting class which contains $K$ we use the following lemma which deals with certain normal products of groups isomorphic to $V \rtimes\langle\omega\rangle$.

Lemma 2.1. Let $H$ be a group and let $A_{1} B_{1}, \ldots, A_{f} B_{f}$ be subgroups of $H$ such that for $i=1, \ldots, f$ :

(i) $A_{i} B_{i} \cong V \rtimes\langle\omega\rangle$, with $A_{i} \cong V$ and $B_{i} \cong\langle\omega\rangle$;

(ii) $A_{i} B_{i} \unlhd H$;

(iii) let $C_{i}=C_{H}\left(A_{i}\right)$ then $B_{i} C_{i} / C_{i}$ is a Sylow q-subgroup of $H / C_{i}$.

Then, for a suitable enumeration, there exists an $e \leq f$ such that

$$
\left\langle A_{1} B_{1}, \ldots, A_{f} B_{f}\right\rangle=A_{1} B_{1} \times \cdots A_{e} B_{e} \times Q,
$$

where $Q$ is a q-group.

Proof. We use induction on $f$. The lemma is obviously true for $f=1$. We now assume

$$
\left\langle A_{1} B_{1}, \ldots, A_{f-1} B_{f-1}\right\rangle=A_{1} B_{1} \times \cdots \times A_{e_{1}} B_{e_{1}} \times Q_{1},
$$


for a suitable $q$-group $Q_{1}$, and $e_{1} \leq f-1$. If $A_{f} B_{f} \cap\left\langle A_{1} B_{1}, \ldots, A_{f-1} B_{f-1}\right\rangle$ $=1$ then $\left\langle A_{1} B_{1}, \ldots, A_{f} B_{f}\right\rangle=A_{f} B_{f} \times\left\langle A_{1} B_{1}, \ldots, A_{f-1} B_{f-1}\right\rangle$ and, apart from reordering the indices, we are finished.

Suppose now that $A_{f} B_{f} \cap\left\langle A_{1} B_{1}, \ldots, A_{f-1} B_{f-1}\right\rangle \neq 1$. Then, since every minimal normal subgroup of $A_{f} B_{f}$ is contained in $A_{f}$, we have $A_{f} \cap\left(A_{1} \times\right.$ $\left.\cdots \times A_{e_{1}}\right) \neq 1$. If $A_{f}$ is not a subgroup of $A_{1} \times \cdots \times A_{e_{1}}$ then, by Maschke's Theorem say, we have $A_{f}=\widehat{A}_{f} \times \widetilde{A}_{f}$, where $\widehat{A_{f}}=A_{f} \cap\left(A_{1} \times \cdots \times A_{e_{1}}\right)$ and $\tilde{A}_{f}$ is a complement to $\widehat{A}_{f}$ which is invariant under $B_{1} \times \cdots \times B_{e_{1}}$. We see that

$$
\left[\tilde{A}_{f}, B_{1} \times \cdots \times B_{e_{1}}\right] \leq \tilde{A}_{f} \cap \hat{A}_{f}=1 .
$$

Now, no element of $A_{1} \times \cdots \times A_{e_{1}}$ is centralised by $B_{1} \times \cdots \times B_{e_{1}}$ so there is some $b \in B_{1} \times \cdots \times B_{e_{1}}$ which does not centralize $\hat{A}_{f}$ (but does centralize $\left.\tilde{A_{f}}\right)$. Since $\langle\omega\rangle$ operates fix-point freely on $V$ we see that $b C_{f} \notin B_{f} C_{f} / C_{f}$. But clearly $B_{f} C_{f} / C_{f}$ is a normal Sylow $q$-subgroup of $H / C_{f}$ (by (iii)), so we have a contradiction. Thus $A_{f} \leq A_{1} \times \cdots \times A_{e_{1}}$. Since $A_{f}=0_{r}\left(A_{f} B_{f}\right)$, we have

$$
\begin{aligned}
A_{f} & =\left[A_{f}, B_{f}\right] \\
& \leq\left[A_{1} \times \cdots \times A_{e_{1}}, B_{f}\right] \\
& =\left[A_{1}, B_{f}\right] \times \cdots \times\left[A_{e_{1}}, B_{f}\right] \\
& =\left(A_{1} \cap A_{f}\right) \times \cdots \times\left(A_{e_{1}} \cap A_{f}\right) .
\end{aligned}
$$

We may suppose that $A_{1} \cap A_{f} \neq 1$. If in addition, say, $A_{2} \cap A_{f} \neq 1$, then we see that

$$
C_{q^{\alpha}} \times C_{q^{\alpha}} \cong B_{1} \times B_{2} \stackrel{\sim}{\leq} H / C_{f},
$$

which again contradicts (iii). Thus we conclude $A_{f}=A_{1}$.

We let $Q_{2}$ be a Sylow $q$-subgroup of $C_{A_{1} B_{1} B_{f}}\left(A_{1}\right)$. By (iii) and comparison of orders we see that $A_{1} B_{1} B_{f}=A_{1} B_{1} \times Q_{2}$. Note that $Q_{2}$ is normal in $H$, since it is characteristic in $A_{1} B_{1} B_{f}$. We let $Q=Q_{1} Q_{2}$ and then have $Q \unlhd H$ and $Q \cap\left\langle A_{1} B_{1}, \ldots, A_{e_{1}} B_{e_{1}}\right\rangle=1$, whence

$$
\left\langle A_{1} B_{1}, \ldots, A_{f} B_{f}\right\rangle=A_{1} B_{1} \times \cdots \times A_{e_{1}} B_{e_{1}} \times Q .
$$

The main steps in the proof of the following result are analogous to those of Construction IV.1 of [4]. It is hoped that the more complicated situation dealt with here will justify the somewhat extreme length of the proof.

CONSTRUCTION 2.2. Let $\mathbf{F}$ be the class of groups which satisfy the following conditions:

(i) $0^{p}\left\{0^{p^{\prime}}(G)\right\}=\left\langle A_{1} B_{1}, \ldots, A_{f} B_{f}, R\right\rangle$, for a suitable $f$ (possibly $\left.f=0\right)$, 
where

(ii) $R=0_{r}\left[0^{p}\left\{0^{p^{\prime}}(G)\right\}\right]$ and, for $i=1, \ldots, f$,

(iii) $A_{i} B_{i} \unlhd 0^{p^{\prime}}(G)$;

(iv) $A_{i} B_{i} \cong V \rtimes\langle\omega\rangle$, with $A_{i} \cong V$ and $B_{i} \cong\langle\omega\rangle$;

(v) let $C_{i}=C_{0^{p^{\prime}}(G)}\left(A_{i}\right)$, then $B_{i} C_{i} / C_{i}$ is a (normal) Sylow q-subgroup of $0^{p^{\prime}}(G) / C_{i}$

(vi) $0_{q}\left[0^{p}\left\{0^{p^{\prime}}(G)\right\}\right]=1$.

Then $\mathbf{F}$ is a Fitting class (which clearly contains $K=V \times\langle\omega, \delta\rangle$ ).

Proof. We first show closure with regard to normal subgroups. Let $G_{1} \unlhd$ $G \in$ F . By [4, II.6],

$0^{p}\left\{0^{p^{\prime}}\left(G_{1}\right)\right\}=\left\langle 0^{p}\left\{0^{p^{\prime}}\left(G_{1}\right)\right\} \cap A_{1} B_{1}, \ldots, 0^{p}\left\{0^{p^{\prime}}\left(G_{1}\right)\right\} \cap A_{f} B_{f}, 0^{p}\left\{0^{p^{\prime}}\left(G_{1}\right)\right\} \cap R\right\rangle$, where $A_{1} B_{1}, \ldots, A_{f} B_{f}$ and $R$ satisfies (i), ., (vi). Note that $R_{1}=$ $0^{p}\left\{0^{p^{\prime}}\left(G_{1}\right)\right\} \cap R=0_{r}\left[0^{p}\left\{0^{p^{\prime}}\left(G_{1}\right)\right\}\right]$. By Lemma 2.1 with (vi), we may assume, without loss of generality, that

$$
\left\langle A_{1} B_{1}, \ldots, A_{f} B_{f}\right\rangle=A_{1} B_{1} \times \cdots \times A_{f} B_{f} .
$$

Now suppose that, say, $A_{1} B_{1} \cap 0^{p}\left\{0^{p^{\prime}}\left(G_{1}\right)\right\} \nless A_{1}$. We see then that there is a $q$-element $b$, say, with $1 \neq b \in A_{1} B_{1} \cap 0^{p}\left\{0^{p^{\prime}}\left(G_{1}\right)\right\}$. By considering the action of $\langle\omega\rangle$ on $V$, we have $A_{1}=\left[A_{1}, b\right] \leq 0^{p}\left\{0^{p^{\prime}}\left(G_{1}\right)\right\}$. Since $0^{p^{\prime}}\left(G_{1}\right)$ is generated by $p$-elements, there must be a $p$-element, $x$, in $0^{p^{\prime}}\left(G_{1}\right)$ such that $x$ does not centralize $B_{1} C_{1} / C_{1}$ for otherwise the factor-group $0^{p^{\prime}}\left(G_{1}\right) /\left(0^{p^{\prime}}\left(G_{1}\right) \cap C_{1}\right)$ (which is isomorphic to $\left.0^{p^{\prime}}\left(G_{1}\right) C_{1} / C_{1}\right)$ would have a non-trivial $q$-factor-group isomorphic to $\langle b\rangle$. Now, since $B_{1}$ is cyclic of order $q^{\alpha}$ (with $q \neq p$ ), we conclude that $A_{1} B_{1}=A_{1}\left[B_{1}, x\right] \leq 0^{p^{\prime}}\left(G_{1}\right)$, whence, in fact, $A_{1} B_{1} \leq 0^{p}\left\{0^{p^{\prime}}\left(G_{1}\right)\right\}$.

We can now assume the $A_{i} B_{i}$ to be ordered such that $\left\langle A_{1} B_{1}, \ldots, A_{d} B_{d}\right\rangle \leq$ $0^{p}\left\{0^{p^{\prime}}\left(G_{1}\right)\right\}$, for some suitable $d$, and $A_{j} B_{j} \cap 0^{p}\left\{0^{p^{\prime}}\left(G_{1}\right)\right\} \leq A_{j} \cap 0^{p}\left\{0^{p^{\prime}}\left(G_{1}\right)\right\}$ $\leq R_{1}$, for $j>d$, and since (vi) is trivially satisfied for $G_{1}$, we conclude that $G_{1} \in \mathbf{F}$.

To show that $\mathrm{F}$ is closed with regard to normal products, we let $G=G_{1} G_{2}$ where $G_{1}$ and $G_{2}$ are normal in $G$ and are elements of $\mathbf{F}$. We let

$$
0^{p}\left\{0^{p^{\prime}}\left(G_{j}\right)\right\}=\left\langle A_{j 1} B_{j 1}, \ldots, A_{j f_{j}} B_{j f_{j}}, R_{j}\right\rangle \text {, for } j=1,2,
$$


where $R_{j}=0_{r}\left[0^{p}\left\{0^{p^{\prime}}\left(G_{j}\right)\right\}\right] \leq 0_{r}\left[0^{p}\left\{0^{p^{\prime}}(G)\right\}\right]$ and $A_{j 1} B_{j 1}, \ldots, A_{j f_{j}} B_{j f_{j}}$ satisfies (iii), .., (vi) in $0^{p^{\prime}}\left(G_{j}\right)$. Again we assume, by Lemma 2.1 that

$$
\left\langle A_{j 1} B_{j 1}, \ldots, A_{j f_{j}} B_{j f_{j}}\right\rangle=A_{j 1} B_{j 1} \times \cdots \times A_{j f_{j}} B_{j f_{j}},
$$

and note that $A_{j 1} B_{j 1} \times \cdots \times A_{j f_{j}} B_{j f_{j}}=0^{q^{\prime}}\left[0^{p}\left\{0^{p^{\prime}}\left(G_{j}\right)\right\}\right]$ is, in particular, a characteristic subgroup of $G_{j}$. We show that, say, $A_{11} B_{11}$ is a normal subgroup of $0^{p^{\prime}}(G)$. Equivalently, we show that $0^{p^{\prime}}\left(G_{2}\right)$ normalises $A_{11} B_{11}$. Note that, by the theorem of Krull-Remak-Schmidt (Huppert [1, p. 69]), if $x \in G$, then either $\left(A_{11} B_{11}\right)^{x}=A_{11} B_{11}$ of $\left(A_{11} B_{11}\right)^{x}$ is one of $A_{12} B_{12}, \ldots, A_{1 f_{1}} B_{1 f_{1}}$.

Now $A_{11} B_{11}$ is subnormal in $\left\langle A_{11} B_{11}, 0_{r}(G)\right\rangle$, is generated by $q$-elements and, by comparison of orders, contains a Sylow $q$-subgroup of the group $\left\langle A_{11} B_{11}, 0_{r}(G)\right\rangle$. Thus $A_{11} B_{11}=0^{q^{\prime}}\left(\left\langle A_{11} B_{11}, 0_{r}(G)\right\rangle\right)$, and so, in particular, $R_{2}$ (which is a subgroup of $0_{r}(G)$ ) normalises $A_{11} B_{11}$.

Suppose, without loss of generality, that $b \in B_{21}$ does not normalize $A_{11} B_{11}$. Since $q>p \geq 3$ we can assume that $\left(A_{11} B_{11}\right)^{b}=A_{12} B_{12}$ and $\left(A_{12} B_{12}\right)^{b}=A_{13} B_{13}$. We let $B_{j i}=\left\langle b_{j i}\right\rangle$ for $j=1,2$ and $i=1, \ldots, f_{j}$. Then we can also assume that $\left(b_{11}\right)^{b}=b_{12}$ and $\left(b_{12}\right)^{b}=b_{13}$. Since $A_{21} B_{21}$ has defect at most two in $0^{p^{\prime}}(G)$, we have $\left[b_{11}, b, b\right]=b_{11} b_{12}^{-2} b_{13} \in A_{21} B_{21}$. From above, $A_{11} \times \cdots \times A_{1 f_{1}}$ normalises $A_{21} B_{21}$, so we have

$$
A_{11} \times A_{12} \times A_{13}=\left[A_{11} \times A_{12} \times A_{13}, b_{11} b_{12}^{-2} b_{13}\right] \leq A_{21} B_{21},
$$

that is, $A_{11} \times A_{12} \times A_{13} \leq A_{21}$, which is a contradiction to the order of $A_{21}$. We conclude that $0^{p}\left\{0^{p^{\prime}}\left(G_{2}\right)\right\}$ normalises $A_{11} B_{11}$.

Finally we let $x$ be a $p$-element of $0^{p^{\prime}}\left(G_{2}\right)$ which does not normalize $A_{11} B_{11}$. Since $p \neq 2$ we again assume that $\left(A_{11} B_{11}\right)^{x}=A_{12} B_{12},\left(A_{12} B_{12}\right)^{x}=$ $A_{13} B_{13},\left(b_{11}\right)^{x}=b_{12}$ and $\left(b_{12}\right)^{x}=b_{13}$ (where the $b_{j i}$ are as above).

Now, $b_{11}^{-1} b_{12}=\left[b_{11}, x\right]$ is a $q$-element of $0^{p^{\prime}}\left(G_{2}\right)$, and so is contained in $0^{p}\left\{0^{p^{\prime}}\left(G_{2}\right)\right\}$. By Sylow's theorems we may assume that

$$
b_{11}^{-1} b_{12} \in\left\langle b_{21}\right\rangle \times \cdots \times\left\langle b_{2 f_{2}}\right\rangle,
$$

so $b_{11}^{-1} b_{12}=\left(b_{21}\right)^{e_{21}} \cdots\left(b_{2 f_{2}}\right)^{e_{2 f_{2}}}$, for suitable powers $e_{2 i}$. By considering the action of $\langle\omega\rangle$ on $V$, we see that for $e_{2 i} \neq \equiv \bmod \left(q^{\alpha}\right)$, we have

$$
A_{2 i}=\left[A_{2 i},\left(b_{2 i}\right)^{e_{2 i}}\right]=\left[A_{2 i}, b_{11}^{-1} b_{12}\right] \leq A_{11} \times A_{12}
$$

(since the $A_{j i} B_{j i}$ have been shown to be normal in $0^{p}\left\{0^{p^{\prime}}(G)\right\}$ ). 
Since we then have $A_{2 i} \leq A_{11} \times A_{12}$, we can have $e_{2 i} \not \equiv 0 \bmod \left(q^{\alpha}\right)$ for at most two values of $i$. Thus we may assume that $b_{11}^{-1} b_{12}=\left(b_{12}\right)^{e_{21}}\left(b_{22}\right)^{e_{22}}$. But then

$$
\begin{aligned}
A_{11} \times A_{12} & =\left[A_{11} \times A_{12}, b_{11}^{-1} b_{12}\right] \\
& =\left[A_{11} \times A_{12}, A_{21} B_{21} \times A_{22} B_{22}\right] \\
& =A_{21} \times A_{22} \quad \text { (by comparison of orders) } .
\end{aligned}
$$

Now $A_{21} \times A_{22}$ is normal in $0^{p^{\prime}}\left(G_{2}\right)$ (since the $A_{2 i}$ are), so we have that $A_{13}=\left(A_{12}\right)^{x} \leq A_{21} \times A_{22}$, whence $A_{11} \times A_{12} \times A_{13} \leq A_{21} \times A_{22}$, which, by comparison of orders, is again a contradiction. We conclude that $0^{p^{\prime}}\left(G_{2}\right)$ normalises $A_{11} B_{11}$, that is, the $A_{j i} B_{j i}$ are normal in $0^{p^{\prime}}(G)$.

We let $C_{11}=C_{0^{p^{\prime}}}\left(A_{11}\right)$ and show that (v) holds for $B_{11} C_{11} / C_{11}$. To do this we first look at the case where there is an element $x \in 0^{p^{\prime}}\left(G_{2}\right)$ such that $\left[A_{11} B_{11}, x\right] \not A_{11}$.

Then, since $A_{11} B_{11} / A_{11} \cong C_{q^{a}}$, we have, in fact, $A_{11} B_{11}=\left[A_{11} B_{11}, x\right] \leq$ $0^{p^{\prime}}\left(G_{2}\right)$. Using commutator arguments as above, we may assume $A_{11} B_{11}=$ $A_{21} B_{21}$ and also $B_{11}=B_{21}$. Now a Sylow $q$-subgroup of $0^{p^{\prime}}(G) / C_{11}$ is contained in $0^{q^{\prime}}\left\{0^{p^{\prime}}(G)\right\} C_{11} / C_{11}$ and

$$
\begin{aligned}
0^{q^{\prime}}\left\{0^{p^{\prime}}(G)\right\} C_{11} / C_{11} & =\left(0^{q^{\prime}}\left\{0^{p^{\prime}}\left(G_{1}\right)\right\} C_{11} / C_{11}\right)\left(0^{q^{\prime}}\left\{0^{p^{\prime}}\left(G_{2}\right)\right\} C_{11} / C_{11}\right) \\
& =\left(\left(B_{11} \times \cdots \times B_{1 f_{1}}\right) C_{11} / C_{11}\right)\left(\left(B_{21} \times \cdots \times B_{2 f_{2}}\right) C_{11} / C_{11}\right) \\
& =\left(B_{11} C_{11} / C_{11}\right)\left(B_{21} C_{11} / C_{11}\right) \\
& =B_{11} C_{11} / C_{11},
\end{aligned}
$$

so $B_{11} C_{11} / C_{11}$ is a Sylow $q$-subgroup of $0^{p^{\prime}}(G) / C_{11}$, as desired.

Now we can assume that $\left[0^{p^{\prime}}\left(G_{2}\right), A_{11} B_{11}\right] \leq A_{11}$. Suppose also that some $q$-element of $0^{p^{\prime}}\left(G_{2}\right)$ does not centralize $A_{11}$. Say, without loss of generality, that $B_{21}$ does not centralize $A_{11}$. Then we have, by normality, $1 \neq\left[A_{11}, B_{21}\right] \leq A_{11} \cap A_{21} B_{21}=A_{11} \cap A_{21}$. Again by considering the action of $\langle\omega\rangle$ on $V$, we see that $C_{B_{11}}\left(A_{11} \cap A_{21}\right)=1$ and $C_{B_{21}}\left(A_{11} \cap A_{21}\right)=1$. We let $\widetilde{C}=C_{0^{p^{\prime}(G)}}\left(A_{11} \cap A_{21}\right)$, and have $A_{11} \leq \widetilde{C}$, so

$$
\left[B_{11} \widetilde{C} / \widetilde{C}, 0^{p^{\prime}}\left(G_{2}\right) \widetilde{C} / \widetilde{C}\right] \leq A_{11} \tilde{C} / \widetilde{C}=1_{0^{p^{\prime}}(G) / \widetilde{C}}
$$

Thus $B_{11} \widetilde{C} / \widetilde{C}$ centralises $0^{p^{\prime}}\left(G_{2}\right) \widetilde{C} / \widetilde{C}$.

Since $\langle\omega\rangle$ acts operator-isomorphically on $V_{1}, \ldots, V_{m}$ (as in Section 1) we see that $A_{11} \cap A_{21}$ can be decomposed as

$$
A_{11} \cap A_{21}=V_{11} \oplus \cdots \oplus V_{1 e}, \text { for some } e \text { with } e \leq m,
$$


where $V_{1 i} \cong U$ (as in Section 1), for $i=1, \ldots, e$ and $V_{1 i}$ and $V_{1 j}$ are operator-isomorphic with regard to $B_{21}$ for all $i$ and $j$ (this follows from (iv)).

Now, $0^{p^{\prime}}\left(G_{2}\right) \widetilde{C} / \widetilde{C}$ is generated by $p$-elements, so there is a $p$-element in $0^{p^{\prime}}\left(G_{2}\right), g$ say, such that $g \widetilde{C}$ does not centralize $B_{21} \widetilde{C} / \widetilde{C}$, and we may assume that $g^{p} \widetilde{C}$ does centralize $B_{21} \widetilde{C} / \widetilde{C}$. We let $\widetilde{V}=A_{11} \cap A_{21}, \tilde{g}=$ $g C,\langle\tilde{\omega}\rangle=B_{21} \widetilde{C} / \widetilde{C}$ and $\langle\tilde{y}\rangle=B_{11} \widetilde{C} / \widetilde{C}$, and we identify $\tilde{g}, \tilde{\omega}$ and $\tilde{y}$ with the elements of $\operatorname{Aut}(\tilde{V})$ which they induce.

Replacing $m$ with $e$, as above, we see that $\langle\tilde{g}, \tilde{\omega}\rangle$ and $\tilde{V}$ satisfy the hypotheses of Lemma 1.4. But then we have a contradiction to Corollary 1.5 , since $\tilde{y}$ centralises $\langle\tilde{g}, \tilde{\omega}\rangle, o(\tilde{y})=q^{\alpha}$ and $C_{\langle\tilde{y}\rangle}(\widetilde{V}) \cong C_{B_{11}}(\widetilde{V})=1$. We conclude that all $q$-elements of $0^{p^{\prime}}\left(G_{2}\right)$ centralize $A_{11}$. Thus the Sylow $q$ subgroup of $0^{p^{\prime}}(G) / C_{11}$ are contained in $0^{p}\left\{0^{p^{\prime}}\left(G_{1}\right)\right\} C_{11} / C_{11}=B_{11} C_{11} / C_{11}$ and again we see that $B_{11} C_{11} / C_{11}$ is the (normal) Sylow $q$-subgroup of $0^{p^{\prime}}(G) / C_{11}$.

We have thus far demonstrated (iii), (iv) and (v) for the $A_{j i} B_{j i}$. Now $0_{q}\left[0^{p}\left\{0^{p^{\prime}}(G)\right\}\right] \leq 0^{q^{\prime}}\left[0^{p}\left\{0^{p^{\prime}}(G)\right\}\right]=\left\langle A_{j i} B_{j i} \mid j=1,2 ; i=1, \ldots, f_{j}\right\rangle$ and we apply Lemma 2.1 to see that

$$
0^{q^{\prime}}\left[0^{p}\left\{0^{p^{\prime}}(G)\right\}\right]=A_{1} B_{1} \times \cdots \times A_{f} B_{f} \times Q,
$$

for a suitable value $f$, where the $A_{d} B_{d}$ are among the $A_{j i} B_{j i}$ and $Q$ is a $q$-group. Clearly $Q=0_{q}\left[0^{p}\left\{0^{p^{\prime}}(G)\right\}\right]$. Since $G_{1}$ and $G_{2}$ are elements of $\mathbf{F}$, we have

$$
Q \cap 0^{p^{\prime}}\left(G_{j}\right)=0_{q}\left[0^{p^{\prime}}\left(G_{j}\right)\right]=0_{q}\left[0^{p}\left\{0^{p^{\prime}}\left(G_{j}\right)\right\}\right]=1,
$$

so $\left[Q, 0^{p^{\prime}}\left(G_{j}\right)\right]=1$ (for $j=1,2$ ), whence $Q \leq Z\left\{0^{p^{\prime}}(G)\right\}$. Now we see that, for $R=0_{r}\left[0^{p}\left\{0^{p^{\prime}}(G)\right\}\right]$,

$$
Q \cap\left\langle A_{1} B_{1} \times \cdots \times A_{f} B_{f}, R\right\rangle=Q \cap\left(A_{1} B_{1} \times \cdots \times A_{f} B_{f}\right)=1,
$$

so, if we let $E=\left\langle A_{1} B_{1} \times \cdots \times A_{f} B_{f}, R\right\rangle$ then we see that $Q E / E$ is in the centre of $0^{p^{\prime}}(G) / E$. In addition $0^{p^{\prime}}(G) / Q E$ is a $p$-group, since $Q E=0^{p}\left\{0^{p^{\prime}}(G)\right\}$. But now $0^{p^{\prime}}(G) / E$ is nilpotent and so has a factor-group isomorphic to $Q$. Since $Q$ is a $p^{\prime}$-group we conclude $Q=1$. Thus

$$
0^{p}\left\{0^{p^{\prime}}(G)\right\}=\left\langle A_{1} B_{1}, \ldots, A_{f} B_{f}, R\right\rangle
$$

and properties (ii), .., (vi) are satisfied. 
By applying the above construction in the respective cases we see that if $K_{1}$ and $K_{2}$ are elements of $\mathbf{K}$, then $K_{1} \in \operatorname{Fit}\left(K_{2}\right)$ if and only if $K_{1} \cong K_{2}$. This type of result seems to indicate that the question as to whether two groups generate the same minimal Fitting class may generally be more easily solved in the case where both groups are extensions of elementary abelian $r$-groups by $r^{\prime}$-linear groups, where the linear groups in question are of nilpotent length greater than or equal to two.

\section{Some Fitting classes which are minimal}

We once more let $p, q, r, \alpha, m$ and $t$ be fixed primes and natural numbers which satisfy (i), .., (vi) of Section 1 . In order to construct some minimal Fitting classes we place two extra conditions on these numbers, namely

(vii) $p^{2} \mathrm{~T}(q-1)$,

(viii) $p \mathrm{~T}|\mathrm{GL}(m, F)|$ (equivalently: $p \mathrm{~T}\left|\mathrm{GL}\left(m, F_{r^{n}}\right)\right|$, where $t=p n$ ), where $F$, as in Section 1, is the field with $r^{l}$ elements. Since $p \neq r$, (viii) requires that $p$ does not divide any of the numbers $r^{t}-1, \ldots, r^{m t}-1$. To reassure ourselves that we are not dealing with the empty set we may check, for example, that the following satisfy conditions (i), .., (viii) : $p=5, q=$ 11, $r=3, \alpha=1, t=5, m=2$ (note: $m=3$ will also do, but not $m=4$ ).

We let the group $K^{*}=V^{*} \rtimes\left\langle\omega^{*}, \delta^{*}\right\rangle$ be constructed in a manner analogous to the group $K$ of Section 1, relative to the above more restricted values of $p, q, r, \alpha, m$, and $t$. We note that, by Lemma 1.1 condition (viii) implies that $p \top\left|C^{*}\right|$, where, analogously to Lemma $1.1, C^{*}=C_{\mathrm{Aut}\left(V^{*}\right)}\left(\omega^{*}\right)$.

We recall that if $G$ is a (finite) group then $\operatorname{Fit}(G)$, the minimal Fitting class which contains $G$ (or Fitting class generated by $G$ ), is

$$
\mathbf{F i t}(G)=\cap\{\mathbf{F}: \mathbf{F} \text { a Fitting class with } G \in \mathbf{F}\} .
$$

We note that it is well known that if $\varepsilon$ is a prime which divides the order of the soluble group $G$, then $\mathbf{S}_{\varepsilon} \subseteq$ Fit $(G)$, where $\mathbf{S}_{\varepsilon}$ is the (Fitting) class of all finite $\varepsilon$-groups.

Construction 3.1. Fit $\left(K^{*}\right)$ is the class of groups which satisfy

(i) $0^{p}(G) \in \operatorname{Fit}\left(V^{*} \rtimes\left\langle\omega^{*}\right\rangle\right)$,

(ii) $0^{p}\left\{0^{p^{\prime}}(G)\right\}=A_{1} B_{1} \times \cdots \times A_{f} B_{f}$, for a suitable $f$ (with possibly $f=0$ ), where, for $i=1, \ldots, f$,

and

(iii) $A_{i} B_{i} \unlhd 0^{p^{\prime}}(G)$

(iv) $A_{i} B_{i} \cong V^{*} \rtimes\left\langle\omega^{*}\right\rangle$, with $A_{i} \cong V^{*}$ and $B_{i} \cong\left\langle\omega^{*}\right\rangle$. 
Proof. We note first that, for the case $m=1$, that is, where $V^{*}$ is irreducible under $\left\langle\omega^{*}\right\rangle$, this result is contained in Bryce [5, Example 5.3]. In addition we easily see that $K^{*}$ is an element of the above class.

The class of groups which satisfy (i) is easily seen to be a Fitting class, so we show that the groups that satisfy (ii), (iii) and (iv) also form a Fitting class. We deal first with normal products. We let $G=G_{1} G_{2}$, where, for $j=1,2, G_{j} \unlhd G$ and such that (ii), (iii) and (iv) hold for $G_{j}$. We see, in particular, that $0^{p}\left\{0^{p^{\prime}}\left(G_{j}\right)\right\}=0^{q^{\prime}}\left[0^{p}\left\{0^{q^{\prime}}\left(G_{j}\right)\right\}\right]$, whence $0^{p}\left\{0^{p^{\prime}}(G)\right\}=$ $0^{q^{\prime}}\left[0^{p}\left\{0^{p^{\prime}}(G)\right\}\right]$.

We now apply Construction 2.2 (relative to the above $p, q, r, \alpha, m$ and $t$ ) and see that, since the groups which satisfy (ii), (iii) and (iv) form a subset of $\mathbf{F}$ (as in Construction 2.2), we have $G \in \mathbf{F}$. Since $0^{p}\left\{0^{p^{\prime}}(G)\right\}$ is generated by $q$-elements, Construction 2.2 also shows that $0^{p}\left\{0^{p^{\prime}}(G)\right\}=$ $\left\langle A_{1} B_{1}, \ldots, A_{f} B_{f}\right\rangle$ for a suitable $f$, where the $A_{i} B_{i}$ satisfy (iii), (iv) and (v) of 11.2. In addition $0_{q}\left[0^{p}\left\{0^{p^{\prime}}(G)\right\}\right]=1$, so by Lemma 2.1, we may assume that $0^{p}\left\{0^{p^{\prime}}(G)\right\}=A_{1} B_{1} \times \cdots \times A_{f} B_{f}$, and this shows that $G$ satisfies (ii), (iii) and (iv) (of Construction 3.1).

This proof is unusual in that closure with regard to normal subgroups is as complicated to demonstrate as closure with regard to normal products. We let $G_{1} \unlhd G$, where $0^{p}\left\{0^{p^{\prime}}(G)\right\}=A_{1} B_{1} \times \cdots \times A_{f} B_{f}$, and (ii), (iii) and (iv) are satisfied. As in the proof of Construction 2.2, we have

$$
0^{p}\left\{0^{p^{\prime}}\left(G_{1}\right)\right\}=\left(0^{p}\left\{0^{p^{\prime}}\left(G_{1}\right)\right\} \cap A_{1} B_{1}\right) \times \cdots \times\left(0^{p}\left\{0^{p^{\prime}}\left(G_{1}\right)\right\} \cap A_{f} B_{f}\right),
$$

and either $A_{i} B_{i} \leq 0^{p}\left\{0^{p^{\prime}}\left(G_{1}\right)\right\}$ or $0^{p}\left\{0^{p^{\prime}}\left(G_{1}\right)\right\} \cap A_{i} B_{i} \leq A_{i}$.

Suppose for some $i$ that $0^{p}\left\{0^{p^{\prime}}\left(G_{1}\right)\right\} \cap A_{i} B_{i} \leq A_{i}$. We then have that $\left[0^{p^{\prime}}\left(G_{1}\right), A_{i} B_{i}\right] \leq A_{i}$, since otherwise, as in the proof of Construction 2.2, we would have, by commutators, that $A_{i} B_{i}$ is a subgroup of $0^{p}\left\{0^{p^{\prime}}\left(G_{1}\right)\right\}$. Thus if $x$ is any $p$-element of $0^{p^{\prime}}\left(G_{1}\right)$ and $\theta_{x}$ is the automorphism of $A_{i}$ induced by conjugation with $x$, then $\theta_{x}$ commutes with $B_{i}$ (considered as a subgroup of $\operatorname{Aut}\left(A_{i}\right)$ ). Since $p T\left|C^{*}\right|$ (for $C^{*}$ as above), we must have $\theta_{x}=$ $1_{\text {Aut }\left(A_{i}\right)}$. Thus $A_{i}$ is centralised by all $p$-elements of $0^{p^{\prime}}\left(G_{1}\right)$ (which is generated by $p$-elements) and we conclude that $0^{p}\left\{0^{p^{\prime}}\left(G_{1}\right)\right\} \cap A_{i} B_{i} \leq Z\left\{0^{p^{\prime}}\left(G_{1}\right)\right\}$.

We put $Z=0^{p}\left\{0^{p^{\prime}}\left(G_{1}\right)\right\} \cap A_{i} B_{i}$, and let

$$
S=0^{p}\left\{0^{p^{\prime}}\left(G_{1}\right)\right\} \cap\left(A_{1} B_{1} \times \cdots \times A_{i-1} B_{i-1} \times A_{i+1} B_{i+1} \times \cdots \times A_{f} B_{f}\right) .
$$

Thus $0^{p}\left\{0^{p^{\prime}}\left(G_{1}\right)\right\}=S \times Z$ and $0^{p^{\prime}}\left(G_{1}\right) /(S \times Z)$ is a $p$-group, so $0^{p^{\prime}}\left(G_{1}\right) / S$ 
is nilpotent. The Sylow $r$-subgroup of $0^{p^{\prime}}\left(G_{1}\right) / S$ is isomorphic to $Z$, so, by nilpotency, $0^{p^{\prime}}\left(G_{1}\right) / S$ has a factor-group isomorphic to $Z$. Since $0^{p^{\prime}}\left(G_{1}\right)$ has no non-trivial $p^{\prime}$-factor-groups, we conclude $Z=1$. For a suitable enumeration of the $A_{i} B_{i}$ we can now assume that

$$
0^{p}\left\{0^{p^{\prime}}\left(G_{1}\right)\right\}=A_{1} B_{1} \times \cdots \times A_{e} B_{e}(e \leq f),
$$

and have verified that $G_{1}$ satisfies (ii), (iii) and (iv).

We denote by $\mathbf{X}$ the class of groups which satisfy (i),.., (iv). Thus $\mathbf{X}$ is a Fitting class which has $K^{*}$ as an element, so $\operatorname{Fit}\left(G^{*}\right) \subseteq \mathbf{X}$. Now let $G$ be any element of $\mathbf{X}$. We show that $G$ is an element of $\operatorname{Fit}\left(K^{*}\right)$. We have $G=0^{p}(G) 0^{p^{\prime}}(G)$, and this is a normal product. By (i), $0^{p}(G) \in$ $\operatorname{Fit}\left(V^{*} \rtimes\left\langle\omega^{*}\right\rangle\right) \subseteq \operatorname{Fit}\left(K^{*}\right)$, so it suffices to show that $0^{p^{\prime}}(G) \in \operatorname{Fit}\left(K^{*}\right)$.

Let $D_{i}=A_{1} B_{1} \times \cdots \times A_{i-1} B_{i-1} \times A_{i+1} B_{i+1} \times \cdots \times A_{f} B_{f}$, for $i=1, \ldots, f$ (where we are taking $G$ to be the group of the statement of the construction). We first show that $0^{p^{\prime}}(G) / D_{i} \in \operatorname{Fit}\left(K^{*}\right)$. For notational convenience we work "modulo" $D_{i}$, that is, we write $D_{i}=1$. We let $P$ be a Sylow $p$-subgroup of $0^{p^{\prime}}(G)$ (so $0^{p^{\prime}}(G)=A_{i} B_{i} P$ ), and write $\widehat{P}=C_{P}\left(A_{i}\right)$. As in the proof of Construction 2.2 , we have $\left[P, A_{i} B_{i}\right]=A_{i} B_{i}$. In addition, considering $B_{i}$ and $P / \widehat{P}$ as the "natural" subgroups of $\operatorname{Aut}\left(A_{i}\right)$, we see, by condition (viii), that no element of $P / \widehat{P}$ centralises $B_{i}$. By condition (vii), $p^{2} \mathrm{~T}(q-1)$, so we conclude that $P / \widetilde{P} \cong C_{p}$. Since $\widehat{P}$ centralises $A_{i}$ we see that there is a decomposition $A_{i} B_{i} \widehat{P}=A_{i} B_{i} \times \widehat{P}$.

We now work modulo $\widehat{P}$. Then $A_{i}$ is self-centralising in $0^{p^{\prime}}(G)$ and $0^{p^{\prime}}(G)$ has a Sylow $p$-subgroup, $\langle x\rangle$, which is cyclic of order $p$. We identify $A_{i}$ with $V^{*}, B_{i}$ with $\left\langle\omega^{*}\right\rangle$ and $\langle x\rangle$ with the "natural" subgroup of $\operatorname{Aut}\left(V^{*}\right)$. Conditions (vii) and (viii) now imply that $\langle x\rangle$ is a Sylow $p$-subgroup of $N^{*}=N_{\text {Aut }\left(V^{*}\right)}\left(\left\langle\omega^{*}\right\rangle\right)$. Thus $\langle x\rangle$ and $\left\langle\delta^{*}\right\rangle$, and hence also $\left\langle\omega^{*}, x\right\rangle$ and $\left\langle\omega^{*}, \delta^{*}\right\rangle$, are conjugate in $N^{*}$. Thus

$$
0^{p^{\prime}}(G) / \widehat{P} \cong V^{*} \rtimes\left\langle\omega^{*}, x\right\rangle \cong V^{*} \rtimes\left\langle\omega^{*}, \delta^{*}\right\rangle=K^{*} .
$$

It follows that

$$
\begin{aligned}
0^{p^{\prime}}(G) & \cong \widetilde{G}=\left\{\left(g A_{i} B_{i}, g \widehat{P}\right) \mid g \in 0^{p^{\prime}}(G)\right\} \\
& \leq\left(0^{p^{\prime}}(G) / A_{i} B_{i}\right) \times\left(0^{p^{\prime}}(G) / \widehat{P}\right) \cong P \times K^{*} .
\end{aligned}
$$

Since $\widetilde{G}$ contains all $p$-elements of $\left(0^{p^{\prime}}(G) / A_{i} B_{i}\right) \times\left(0^{p^{\prime}}(G) / \widehat{P}\right)$, it is subnormal in the latter group. Thus $0^{p^{\prime}}(G)$ is isomorphic to a subnormal subgroup 
of $P \times K^{*}$. Since $P$ is a $p$-group, the remark preceding this construction shows that $P \in \operatorname{Fit}\left(K^{*}\right)$. We conclude that $0^{p^{\prime}}(G) / D_{i} \in \operatorname{Fit}\left(K^{*}\right)$.

Since $\bigcap_{i=1}^{f} D_{i}=1$, we see that $0^{p^{\prime}}(G)$ is isomorphic to

$$
\left\{\left(g D_{1}, \ldots, g D_{f}\right) \mid g \in 0^{p^{\prime}}(G)\right\}
$$

which, as above, is subnormal in $\left(0^{p^{\prime}}(G) / D_{1}\right) \times \cdots \times\left(0^{p^{\prime}}(G) / D_{f}\right)$. Thus $0^{p^{\prime}}(G)$ is isomorphic to a subnormal subgroup of an element of $\operatorname{Fit}\left(K^{*}\right)$, and so $G \in \operatorname{Fit}\left(K^{*}\right)$ and $X=\operatorname{Fit}\left(K^{*}\right)$.

\section{Concluding remarks}

One major difference between the Fitting classes $F_{1}$, say, of Construction 2.2 and $\mathbf{F}_{2}$ of Construction 3.1 is that $\mathbf{S}_{r} \mathbf{S}_{p} \subseteq \mathbf{F}_{1}$, while $\mathbf{S}_{r} \mathbf{S}_{p} \cap \mathbf{F}_{2}=\mathbf{N}_{\{p, r\}}$, where $\mathbf{S}_{r} \mathbf{S}_{p}$ is the class of all (finite) extensions of $r$-groups by $p$-groups and $\mathbf{N}_{\{p, r\}}$ is the class of all nilpotent $\{p, r\}$-groups, for the given values of $p$ and $r$. Thus the extra conditions (vii) and (viii) of Section 3 yield more precise information about the $\{p, r\}$-groups in $\operatorname{Fit}\left(K^{*}\right)$.

To show that the latter is not always the case, we let $p, q, r, \alpha, m$ and $t$ satisfy (i), .., (vi) of Section 1, and add in the new condition

(vii) $^{\prime} p \| \mathrm{GL}(m, F) \mid$

(in contrast to (viii) of Section 3). We can take $p=5, q=11, r=3, \alpha=$ $1, t=5$ and $m=4$, to see that such numbers $d o$ exist. We let $\widehat{K}=$ $\widehat{V} \rtimes\langle\hat{\omega}, \hat{\delta}\rangle$ be constructed as in Section 1, relative to the above numbers.

Proposition 4.1. $\mathbf{F i t}(\hat{K}) \cap \mathbf{S}_{r} \mathbf{S}_{p} \nsubseteq \mathbf{N}_{\{p, r\}}$ (that is, there are non-nilpotent $\{p, r\}$-groups in $\operatorname{Fit}(\widehat{K}))$.

Proof. We let $s$ and $\beta$ be defined as in Section 1. Thus, by (vii) ${ }^{\prime}, s \neq 0$ and $\beta \geq 1$. We let $\hat{h}_{1}$ be defined analogously to $h_{1}$ of Section 1 . Thus $\hat{h}_{1}$ centralises $\hat{\omega}$ in $\operatorname{Aut}(\hat{V})$ and $\hat{\delta}$ normalises $\left\langle\hat{h}_{1}\right\rangle$. We form the semidirect product $\widehat{G}=\hat{V} \rtimes\left\langle\hat{\omega}, \hat{\delta}, \hat{h}_{1}\right\rangle$. Since $0^{p^{\prime}}(\widehat{G})=\widehat{V} \rtimes\langle\hat{\omega}\rangle \leq \widehat{K}$, we see that $\widehat{K}$ is subnormal in $\widehat{G}$. Since Fitting classes are known to be closed with regard to subnormal products, we see that $(\widehat{K})^{\widehat{G}} \in \mathbf{F i t}(\widehat{K})$ (where $(\widehat{K})^{\widehat{G}}$ is the normal closure of $\widehat{K}$ in $\widehat{G}$ ).

We define $\widehat{W}_{1}$ analogously to $W_{1}$ in Section 1 . Considering the restrictions to $\widehat{W}_{1}$, we see by Lemma 1.3 , that $\widehat{W}_{1}$ is irreducible under $\left\langle\hat{\omega} \hat{h}_{1}\right\rangle$. As in [3, III.3], say, we may identify $\widehat{W}_{1}$ with the additive group of $F_{r^{t}}$, the 
field with $r^{s t}$ elements. We also identify $\hat{\omega} \hat{h}_{1}$ with an element of order $p^{\beta} q^{\alpha}$ in the multiplicative group of $F_{r^{s t}}$ and $\hat{\delta}$ with the Galois automorphism of order $p$. Letting $n$ be such that $t=n p$, and $F_{r^{s}}$ be the subfield of order $r^{s n}$ which comprises those elements fixed by the action of $\hat{\delta}$, we see by, say [3, III.4], that $\hat{h}_{1} \notin F_{r^{s n}}$. Thus $\hat{\delta}$ does not centralize $\hat{h}_{1}$.

We let $\hat{c}=\left[\hat{\delta}, \hat{h}_{1}\right]$. Then $1 \neq \hat{c} \in(\widehat{K})^{\widehat{G}} \cap\left\langle\hat{h}_{1}\right\rangle$. Since $\langle\hat{c}\rangle$ is characteristic in $\left\langle\hat{h}_{1}\right\rangle$, which is normal in $\left\langle\hat{\omega}, \hat{\delta}, \hat{h}_{1}\right\rangle$, we have that $\widehat{V} \rtimes\langle\hat{c}\rangle \unlhd(\widehat{K})^{\widehat{G}} \in$ $\operatorname{Fit}(\widehat{K})$. Thus $\widehat{V} \times\langle\hat{c}\rangle$ is a non-nilpotent group which is an element of $\mathbf{S}_{r} \mathbf{S}_{p} \cap$ $\operatorname{Fit}(\widehat{K})$.

We finally indicate why condition (vi) (that is $1 \leq m<p$ ) of Section 1 is necessary in order to construct Fitting classes like those in Construction 2.2. We let $G$ be a subgroup of $\mathrm{GL}(e, K)$, where $K$ is any field, and define the tensor-products

$$
I_{e} \otimes G=\left\{\left(\begin{array}{lll}
g & & 0 \\
& \ddots & \\
0 & & g
\end{array}\right) \mid g \in G\right\},
$$

and

$$
G \otimes I_{e}=\left\{\left(\begin{array}{ccc}
g_{11} I_{e} & \cdots & g_{1 e} I_{e} \\
\vdots & & \vdots \\
g_{e 1} I_{e} & \cdots & g_{e e} I_{e}
\end{array}\right) \mid\left(\begin{array}{ccc}
g_{11} & \cdots & g_{1 e} \\
\vdots & & \vdots \\
g_{e 1} & \cdots & g_{e e}
\end{array}\right) \in G\right\},
$$

(where $I_{e}$ is the $e \times e$ identity matrix). Thus both tensor-products are groups of $e^{2} \times e^{2}$ matrices which are isomorphic to $G$.

Proposition 4.2. (i) $I_{e} \otimes G$ and $G \otimes I_{e}$ commute elementwise in $\mathrm{GL}\left(e^{2}, K\right)$, (ii) $I_{e} \otimes G$ and $G \otimes I_{e}$ are conjugate in $\operatorname{GL}\left(e^{2}, K\right)$.

Proof. (i) is easy to verify directly. For (ii), we write $\widetilde{G}=I_{e} \otimes G$ and let $\widetilde{G}$ act operator-isomorphically, with its "natural" action, on the vectorspaces $U_{1}, \ldots, U_{e}$, all of dimension $e$ over $K$, where $u_{i 1}, \ldots, u_{i e}$ is a basis of $U_{i}(i=1, \ldots, e)$ which induces the given representation of $G$. We let $U=U_{1} \oplus \cdots \oplus U_{e}$, that is, $U$ is the direct sum of $e$ operatorisomorphic $\widetilde{G}$-submodules. We choose a new basis of $U$ by letting $w_{i j}=u_{j i}$ $i, j=1, \ldots, e$.

We can check that the action of $\tilde{G}$ with respect to the $w_{i j}$ is represented by $G \otimes I_{e}$. Thus $\widetilde{G}$ and $G \otimes I_{e}$ are equivalent representations and hence conjugate in $\mathrm{GL}\left(e^{2}, K\right)$. 
We let $\left\langle\omega_{1}, \delta_{1}\right\rangle$ be as in Section 1 and, letting $F_{r^{n}}$ be the subfield of order $r^{n}$ in $F$ (as in Section 1, where $t=n p$ ), we see that $F_{r^{n}}$ is centralised by the action of $\delta_{1}$. We can thus consider $\left\langle\omega_{1}, \delta_{1}\right\rangle$ to be represented over $F_{r^{n}}$, that is, $\left\langle\omega_{1}, \delta_{1}\right\rangle \leq \mathrm{GL}\left(p, F_{r^{n}}\right)$.

We let

$$
\begin{aligned}
& \widetilde{G}=I_{p} \otimes\left\langle\omega_{1}, \delta_{1}\right\rangle \leq \mathrm{GL}\left(p^{2}, F_{r^{n}}\right), \\
& \widehat{G}=\left\langle\omega_{1}, \delta_{1}\right\rangle \otimes I_{p} \leq \mathrm{GL}\left(p^{2}, F_{r^{n}}\right)
\end{aligned}
$$

and we let $\langle\tilde{\omega}\rangle=I_{p} \otimes\left\langle\omega_{1}\right\rangle$. We identify $\operatorname{GL}\left(p^{2}, F_{r^{n}}\right)$ with the "natural" subgroup of $\mathrm{GL}\left(p^{2} n, r\right)=\mathrm{GL}(p t, r)$. By Proposition $4.2 \widetilde{G}$ and $\widehat{G}$ are conjugate and centralize each other in $\operatorname{GL}(p t, r)$. Since $Z\left(\left\langle\omega_{1}, \delta_{1}\right\rangle\right)=1$, we have that $\langle\widetilde{G}, \widehat{G}\rangle=\widetilde{G} \times \widehat{G}$. Now let $\widetilde{V}$ be the direct sum $\widetilde{V}=U_{1} \oplus \cdots \oplus U_{p}$, where $U_{i} \cong U$ (as in Section 1), for $i=1, \ldots, p$, and let $\widetilde{K}=\widetilde{V} \rtimes \widetilde{G}$. Thus $\widetilde{K}$ is constructed analogously to $K$ of Section 1 , only with $m=p$.

From the above considerations $\widetilde{K}$ and $\widetilde{V} \times \widehat{G}$ are isomorphic and also $\widetilde{V} \rtimes(\widetilde{G} \times \widehat{G})$ is the normal product of $\widetilde{K}$ and $\widetilde{V} \rtimes \widehat{G}$. So $\widetilde{V} \rtimes(\widetilde{G} \times \widehat{G}) \in \operatorname{Fit}(\widetilde{K})$. By considering condition (v) of Construction 2.2, we see that a Fitting class construction directly analogous to that of Construction 2.2 , with $\widetilde{V} \rtimes\langle\tilde{\omega}\rangle$ in place of $V \times\langle\omega\rangle$, is not possible.

\section{References}

[1] B. Huppert, Endliche Gruppen I, (Berlin-Heidelberg-New York 1967),

[2] B. McCann, 'A Fitting class construction' (to appear).

[3] B. McCann, 'Examples of normal products of finite groups and an application to Fitting classes' (submitted).

[4] B. McCann, 'Examples of minimal Fitting classes of finite groups', Arch. Math. 49 (1987), 179-186.

[5] R. A. Bryce, The Fitting Class Generated by a Finite Soluble Group, (Research Report No. 34-1988, A.N.U. Mathematics Research Report Series).

[6] R. A. Bryce, J. Cossey and E. Ormerod, 'Fitting classes after dark', Proceedings of the Singapore Group Theory Conference 1987, pp. 293-321 (de Gruyter, Berlin-New York, 1989).

[7] R. S. Dark, 'Some examples in the theory of injectors of finite soluble groups', Math. Z. 27 (1972), 145-156.

Department of Physical and Quantitative Sciences

Regional Technical College

Waterford

Ireland 